\title{
Exact Solution for Pressure Driven Flow of Two Immiscible Phan-Thien-Tanner Fluids in a Pipe
}

\author{
A. M. Siddiqui' ${ }^{1}$, A. Walait ${ }^{2}$, T. Allison'1, T. Haroon ${ }^{1}$ \\ ${ }^{1}$ Deparment of Mathematics, Pennsylvania State University, York Campus, York, PA, USA \\ ${ }^{2}$ Department of Mathematics, Riphah International University Islamabad Campus, Pakistan \\ Email: tahirapak@yahoo.com
}

How to cite this paper: Siddiqui, A.M., Walait, A., Allison, T. and Haroon, T. (2018) Exact Solution for Pressure Driven Flow of Two Immiscible Phan-Thien-Tanner Fluids in a Pipe. Open Journal of Fluid Dynamics, 8, 378-391.

https://doi.org/10.4236/ojfd.2018.84024

Received: August 16, 2018

Accepted: November 5, 2018

Published: November 8, 2018

Copyright () 2018 by authors and Scientific Research Publishing Inc. This work is licensed under the Creative Commons Attribution International License (CC BY 4.0).

http://creativecommons.org/licenses/by/4.0/

\begin{abstract}
This paper provides the exact solutions for the fully developed two layer pressure driven flows of incompressible Phan-Thien-Tanner fluids in a horizontal cylindrical pipe. Exact equations are formulated and solved for important kinematic properties, such as, velocity profiles, normal and shear stresses, total volume fluxes through a circular cross-section and average velocities. Graphical results are provided and discussed for the different flow parameters. A comparison of Upper Convected Maxwell (UCM), Linear Phan-Thien-Tanner (LPTT) and Exponential Phan-Thien-Tanner (EPTT) shows that UCM is a low viscosity fluid as compared to LPTT, and EPTT and LPTT is lighter than EPTT.
\end{abstract}

\section{Keywords}

Exact Solution, Two-Layer Flow, PTT Fluids

\section{Introduction}

The Navier-Stokes equations governing the motion of the viscous fluid are non-linear and their exact solutions are rare or non-existent. The nonlinearity in these equations is caused by the presence of convected term and in the case of non-Newtonian fluids, the use of constitutive equations. Because of the great variety in physical structure of non-Newtonian fluids, it is not possible to describe their mechanical behavior by a single constitutive equation. For this reason, a great variety of constitutive equations have been proposed [1]. Among many equations, the constitutive equations proposed by Phan-Thien and Tanner [2] [3] have been the subject of increasingly extensive study in recent years. Oliveira and Pinho [4] studied the problem of fully developed channel and pipe flows of PTT fluids and obtained an analytical expression for velocity fields and stress 
components in both geometries. Some other studies regarding PTT have been carried out in [5] [6] [7]. Letelier and Siginer [8] studied the problem of fully developed pipe flow of a class of nonlinear viscoelastic fluids which include PTT and Johnson-Segalman models as special cases. Some other works using PTT fluid have been carried out by Siddiqui et al. in [9]. All these studies are based on the flow of a single PTT fluid. This paper however, deals with a two-layer concentric flow of two immiscible PTT fluids in a single pipe.

Recently, the interest in stratified laminar flow of two or more immiscible fluids has grown considerably because of their wide use in technological processes. There has been some theoretical and experimental work on the stratified laminar flow of two immiscible fluids in a horizontal pipe. For instance, Packham and Shall [10] studied the stratified laminar flow of two immiscible fluids in a pipe, Brauner [11] analyzed the annular-core flow of two immiscible liquids, and Kapur and Shulka [12] investigated the flow of $n$ layers of immiscible fluids of different heights between two plates and had shown that for any number of fluids, ignoring their heights, a unique maximum velocity always exists. The reason for the interest in this configuration stems from the idea of possibly reducing the power required to pump oil in a pipeline by the addition of water. The combination of two melt streams (co-extrusion through a die) has become a very economical method of producing materials with unique properties in polymer processing. All the above mentioned works on multi-layered flows of immiscible fluids have been carried out on Newtonian fluids. This paper deals with the investigation of flow of two layers of different immiscible PTT fluids. Since PTT fluids are viscoelastic in nature, the work for this model represents a variety of industrial applications very well.

In this paper mathematics reveals a need for a system of four first-order linear ordinary differential equations associated with four inhomogeneous boundary conditions. We will solve this system of differential equations simultaneously, including the boundary conditions, in order to calculate the velocity profiles, total volume fluxes and the average velocities resulting from the flow UCM, LPTT and EPTT models.

\section{Governing Equations}

The basic equations governing the isothermal two-layer flow of immiscible incompressible fluids are the continuity and momentum equations. Mathematically,

$$
\begin{gathered}
\operatorname{div} \boldsymbol{V}^{(k)}=0 ; \quad k=1,2, \\
\rho^{(k)} \frac{D \boldsymbol{V}^{(k)}}{D t}=-\nabla p+\nabla \cdot \boldsymbol{T}^{(k)}+\rho^{(k)} \boldsymbol{b} ; k=1,2,
\end{gathered}
$$

where the superscript $k$ denotes the two fluids, the number $k=1$ represents the fluid in the core, while the fluid properties for the outer fluid along the wall are denoted by the superscript $k=2 . \boldsymbol{V}^{(k)}, \rho^{(k)}, \boldsymbol{T}^{(k)}$ are the velocity, density 
and extra stress tensor of the $k$ fluid respectively. $\frac{D}{D t}$ is the material derivative defined as

$$
\frac{D(\star)}{D t}=\frac{\partial(\star)}{\partial t}+(\boldsymbol{V} \cdot \nabla)(\star) .
$$

The general form of the constitutive equation defining the PTT fluid is

$$
f\left(\operatorname{tr}\left(\boldsymbol{T}^{(k)}\right)\right) \boldsymbol{T}^{(k)}+\lambda^{(k)} \stackrel{\nabla^{(k)}}{\boldsymbol{T}}=\eta^{(k)} \boldsymbol{A}_{1}^{(k)} ; \quad k=1,2,
$$

where $\eta^{(k)}, \lambda^{(k)}$ and $\operatorname{tr}\left(\boldsymbol{T}^{(k)}\right)$ are the viscosity, relaxation time and trace of stress tensor of the $k$ fluid and Rivlin-Ericksen tensor $A_{1}^{(k)}$ given by

$$
\boldsymbol{A}_{1}^{(k)}=\nabla \boldsymbol{V}^{(k)}+\left(\nabla \boldsymbol{V}^{(k)}\right)^{\mathrm{T}} ; \quad k=1,2,
$$

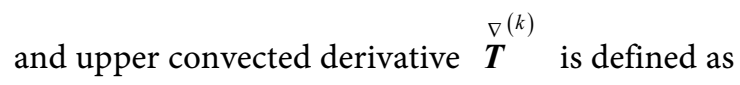

$$
\stackrel{\nabla}{(k)}^{\left(\frac{D \boldsymbol{T}^{(k)}}{D t}\right.}-\left(\boldsymbol{T}^{(k)}\left(\nabla \boldsymbol{V}^{(k)}\right)+\left(\nabla \boldsymbol{V}^{(k)}\right)^{\mathrm{T}} \boldsymbol{T}^{(k)}\right) ; \quad k=1,2 .
$$

The three PTT models, commonly used are

$$
\begin{gathered}
f\left(t r \boldsymbol{T}^{(k)}\right)=1, \quad \text { (UCM Model), } \\
f\left(\operatorname{tr} \boldsymbol{T}^{(k)}\right)=1+\frac{\epsilon^{(k)} \lambda^{(k)}}{\eta^{(k)}} \operatorname{tr}\left(\boldsymbol{T}^{(k)}\right), \quad \text { (LPTT Model), } \\
f\left(t r \boldsymbol{T}^{(k)}\right)=\exp \left(\frac{\epsilon^{(k)} \lambda^{(k)}}{\eta^{(k)}} \operatorname{tr}\left(\boldsymbol{T}^{(k)}\right)\right), \quad \text { (EPTT Model), }
\end{gathered}
$$

where $\epsilon^{(k)}$ is a parameter related to the elongation behavior of the model. In the absence of the parameters $\epsilon^{(k)}$, the models (7) and (8) reduce to the well known Maxwell model (6).

\section{Problem Formulation}

We consider two-layer immiscible flow of incompressible PTT fluids in a horizontal cylindrical pipe of radius $R_{2}$ as shown in Figure 1. The interface of the two fluids lies at a constant distance $R_{1}$ from the axis of the pipe. To visualize the problem a cylindrical coordinate system is chosen, where the axis of the pipe will be denoted by $z$, while $r$ will denote the radially outward measured distance from the $z$-axis. The flow is driven by the constant pressure gradient, assuming that the flow is steady, unidirectional and fully developed.

The velocities and extra stresses can be taken of the form

$$
\boldsymbol{V}^{(k)}=\left[0,0, w^{(k)}(r)\right], \quad \boldsymbol{T}^{(k)}=\boldsymbol{T}^{(k)}(r) ; \quad k=1,2,
$$

where $w$ is the axial velocity component. Using Equation (9) in Equations (3)-(5), non-zero stress components become

$$
f\left(t r \boldsymbol{T}^{(k)}\right) T_{r z}^{(k)}=\eta^{(k)} \frac{\mathrm{d} w^{(k)}}{\mathrm{d} r} ; \quad k=1,2,
$$




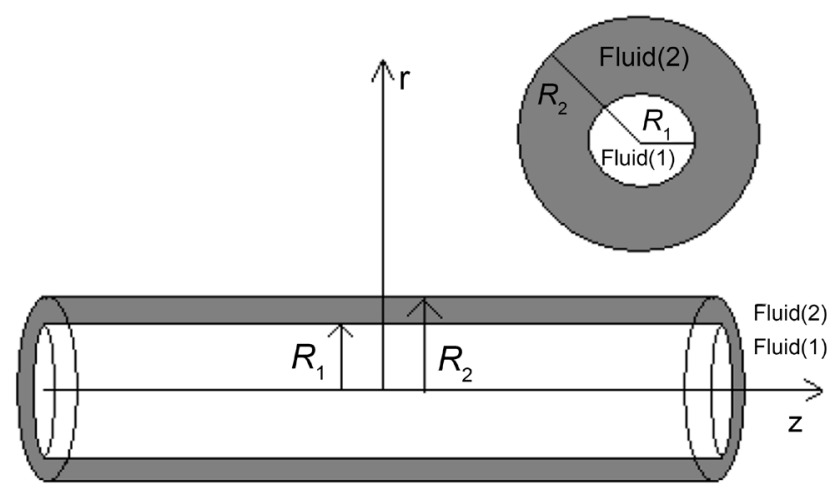

Figure 1. Geometry of the problem.

$$
f\left(t r \boldsymbol{T}^{(k)}\right) T_{r r}^{(k)}=2 \lambda^{(k)} T_{r z}^{(k)} \frac{\mathrm{d} w^{(k)}}{\mathrm{d} r} ; \quad k=1,2 .
$$

Solving Equation (10) and Equation (11) simultaneously, we obtain

$$
T_{r r}^{(k)}=\frac{2 \lambda^{(k)} T_{r z}^{(k) 2}}{\eta^{(k)}} ; \quad k=1,2 .
$$

Trace of stress tensor of $k$ th PTT fluid is given by

$$
\operatorname{tr}\left(\boldsymbol{T}^{(k)}\right)=T_{r r}^{(k)}+T_{\theta \theta}^{(k)}+T_{z z}^{(k)}=\frac{2 \lambda^{(k)} T_{r z}^{(k) 2}}{\eta^{(k)}} ; \quad k=1,2 .
$$

Using Equation (9) in Equation (1), the continuity equation is identically satisfied and the non-zero component of the momentum Equation (2) becomes

$$
\frac{1}{r} \frac{\partial}{\partial r}\left(r T_{r z}^{(k)}\right)=-C ; \quad k=1,2,
$$

where $\left(-\frac{\partial p}{\partial z}\right)=C$. The boundary conditions for the problem under consideration are given by

$$
\begin{gathered}
T_{r z}^{(1)}=0, \quad \text { at } r=0, \\
T_{r z}^{(1)}=T_{r z}^{(2)}, \quad \text { at } r=R_{1}, \\
w^{(1)}=w^{(2)}, \quad \text { at } r=R_{1}, \\
w^{(2)}=0, \quad \text { at } r=R_{2} .
\end{gathered}
$$

We note here that these conditions are four in number and are sufficient to find the solution to our problem which consists of four first order differential equations, two first order differential equations represented by Equation (14) and two differential equations obtained for velocity by simplifying either Equation (10) or Equation (11). Hence, our problem is consistent and we attempt to find exact solutions to the problem.

\section{Solution of The Problem}

Equation (14), upon integration with respect to $r$, yields 


$$
r T_{r z}^{(k)}=-C \frac{r^{2}}{2}+A^{(k)}, \quad k=1,2
$$

where $A^{(k)}, k=1,2$, are constants of integration. Using the condition (15) in the equation (19) when $k=1$, we find that $A^{(1)}=0$, so that we obtain

$$
T_{r z}^{(1)}=-C \frac{r}{2} ; \quad 0 \leq r \leq R_{1}
$$

Similarly, Equation (19) with the help of boundary condition (16) gives $A^{(2)}=0$ which yields

$$
T_{r z}^{(2)}=-C \frac{r}{2} ; \quad R_{1} \leq r \leq R_{2} .
$$

The expressions (20) and (21) clearly indicate that the shear stresses of both the fluids are the same and vary linearly with the distance from the axis of the pipe, which implies

$$
T_{r z}^{(k)}=-C \frac{r}{2} ; \quad k=1,2,
$$

for which we see that for fluid (1) and fluid (2) the regions are $0 \leq r \leq R_{1}$ and $R_{1} \leq r \leq R_{2}$, respectively.

This last result when used in Equation (12) for normal stresses leads to

$$
T_{r r}^{(k)}=\frac{\lambda^{(k)} C^{2}}{2 \eta^{(k)}} r^{2} ; \quad k=1,2 .
$$

Thus the normal stresses for both the fluids are different, depending on their corresponding material constants. Moreover, these normal stresses increase with the square of the distance from the axis of the tube. For Newtonian fluids, $\lambda^{(k)}=0$ and we find that the shear stresses remain unchanged while the normal stresses are zero.

Trace of extra stress tensor is given by

$$
\operatorname{tr}\left(\boldsymbol{T}^{(k)}\right)=\frac{\lambda^{(k)} C^{2}}{2 \eta^{(k)}} r^{2} ; \quad k=1,2 .
$$

Velocity profile can be calculated by rewriting in simplified form equation (10) resulting in the equation

$$
\frac{\mathrm{d} w^{(k)}}{\mathrm{d} r}=\frac{f\left(\operatorname{tr} \boldsymbol{T}^{(k)}\right) T_{r z}^{(k)}}{\eta^{(k)}} ; \quad k=1,2
$$

which is a system of first order non-homogeneous linear ordinary differential equations for two immiscible PTT fluids.

\subsection{Solution for the Upper Convected Maxwell Model}

By making use of expressions (6) and (22) in expression (25), we get

$$
\frac{\mathrm{d} w_{u}^{(k)}}{\mathrm{d} r}=-\frac{C}{2 \eta^{(k)}} r, \quad k=1,2,
$$

where subscript $u$ stands for UCM model. By integrating Equation (26), we get 
velocity profiles for fluid (1) and fluid (2), with two constants of integration and those constants are evaluated by using boundary conditions (18) and (17). Velocity profiles for both immiscible fluid are given by

$$
\begin{aligned}
& w_{u}^{(1)}=C\left(\frac{R_{1}^{2}-r^{2}}{4 \eta^{(1)}}+\frac{R_{2}^{2}-R_{1}^{2}}{4 \eta^{(2)}}\right) ; \quad 0 \leq r \leq R_{1}, \\
& w_{u}^{(2)}=C\left(\frac{R_{2}^{2}-r^{2}}{4 \eta^{(2)}}\right) ; \quad R_{1} \leq r \leq R_{2} .
\end{aligned}
$$

Flow rates through a circular cross section of a pipe bounded in the region $0 \leq r \leq R_{1}$ and $R_{1} \leq r \leq R_{2}$ are given by

$$
Q_{u}^{(1)}=2 \pi \int_{0}^{R_{1}} w_{u}^{(1)}(r) r \mathrm{~d} r, \quad Q_{u}^{(2)}=2 \pi \int_{R_{1}}^{R_{2}} w_{u}^{(2)}(r) r \mathrm{~d} r .
$$

We use the expression for velocity profiles (27) in formulas (28) and obtain

$$
\begin{aligned}
& Q_{u}^{(1)}=\pi R_{1}^{2} C\left(\frac{R_{2}^{2}-R_{1}^{2}}{4 \eta^{(2)}}+\frac{R_{1}^{2}}{8 \eta^{(1)}}\right), \\
& Q_{u}^{(2)}=\pi C\left(\frac{R_{2}^{4}+R_{1}^{4}-2 R_{1}^{2} R_{2}^{2}}{8 \eta^{(2)}}\right) .
\end{aligned}
$$

Net volume flux through the circular pipe of radius $R_{2}$ is given by

$$
Q_{u}=\frac{\pi C}{8}\left(\frac{R_{2}^{4}-R_{1}^{4}}{\eta^{(2)}}+\frac{R_{1}^{4}}{\eta^{(1)}}\right) .
$$

Average velocity is given by

$$
\bar{v}_{u}=\frac{Q_{u}}{\pi R_{2}^{2}} .
$$

Substituting expression (31), average velocity becomes

$$
\bar{v}_{u}=\frac{C}{8 R_{2}^{2}}\left(\frac{R_{2}^{4}-R_{1}^{4}}{\eta^{(2)}}+\frac{R_{1}^{4}}{\eta^{(1)}}\right),
$$

Above results are same as for the case of two immiscible Newtonian fluids with viscosities $\eta^{(1)}$ and $\eta^{(2)}$.

\subsection{Solution for the Linear Phan-Thien-Tanner (LPTT) Model}

By using trace of extra stress tensor (24) in linear model (7) and then using that model and shear stresses (22), in differential Equation (25), we get

$$
\frac{\mathrm{d} w_{l}^{(k)}}{\mathrm{d} r}=-\frac{C}{2 \eta^{(k)}} r-\frac{C^{3} \epsilon^{(k)} \lambda^{(k) 2}}{4 \eta^{(k) 3}} r^{3}, \quad k=1,2,
$$

where subscript $l$ stands for LPTT fluid model. Integrating with respect to $r$ we get

$$
w_{l}^{(k)}=-\frac{C}{4 \eta^{(k)}} r^{2}-\frac{C^{3} \epsilon^{(k)} \lambda^{(k) 2}}{16 \eta^{(k) 3}} r^{4}+B_{k}, \quad k=1,2,
$$


where $B_{k}, k=1,2$ are the constants of integration. For $k=2$, the Equation (34) with the boundary condition (18), yields

$$
w_{l}^{(2)}=\frac{C}{4 \eta^{(2)}}\left(R_{2}^{2}-r^{2}\right)+\frac{C^{3} \epsilon^{(2)} \lambda^{(2) 2}}{16 \eta^{(2) 3}}\left(R_{2}^{4}-r^{4}\right) ; \quad R_{1} \leq r \leq R_{2} .
$$

with the help of the expression (36) for $w_{l}^{(2)}$ and the boundary condition (17) the Equation (35) for $k=1$, gives

$$
\begin{aligned}
& w_{l}^{(1)}=\frac{C}{4}\left[\frac{\left(R_{1}^{2}-r^{2}\right)}{\eta^{(1)}}+\frac{\left(R_{2}^{2}-R_{1}^{2}\right)}{\eta^{(2)}}\right] \\
& +\frac{C^{3}}{16}\left[\frac{\epsilon^{(1)} \lambda^{(1) 2}}{\eta^{(1) 3}}\left(R_{1}^{4}-r^{4}\right)+\frac{\epsilon^{(2)} \lambda^{(2) 2}}{\eta^{(2) 3}}\left(R_{2}^{4}-R_{1}^{4}\right)\right] ; \quad 0 \leq r \leq R_{1} .
\end{aligned}
$$

Flow rates for both the fluids are obtained by using Equation (36) and Equation (37) in Equation (28) as

$$
\begin{gathered}
Q_{l}^{(1)}=\pi C R_{1}^{2}\left\{\frac{R_{2}^{2}-R_{1}^{2}}{4 \eta^{(2)}}+\frac{R_{1}^{2}}{8 \eta^{(1)}}\right\}+\frac{\pi C^{3} R_{1}^{2}}{8}\left\{\frac{\epsilon^{(2)} \lambda^{(2) 2}}{2\left(\eta^{(2)}\right)^{3}}\left(R_{2}^{4}-R_{1}^{4}\right)+\frac{\epsilon^{(2)} \lambda^{(1) 2}}{3\left(\eta^{(1)}\right)^{3}} R_{1}^{4}\right\}, \\
Q_{l}^{(2)}=\pi C\left\{\frac{R_{1}^{4}+R_{2}^{4}-2 R_{2}^{2} R_{1}^{2}}{8 \eta^{2}}\right\}+\frac{\pi C^{3} \epsilon^{(2)} \lambda^{(2) 2}}{48 \eta^{(2) 3}}\left\{2 R_{2}^{6}+R_{1}^{6}-3 R_{2}^{4} R_{1}^{2}\right\} .
\end{gathered}
$$

The total volume flux $Q_{l}$ through the circular cross section of the pipe of radius $R_{2}$ for two immiscible LPTT fluids is obtained as

$$
\begin{aligned}
Q_{l} & =Q_{l}^{(1)}+Q_{l}^{(2)} \\
& =\frac{\pi C}{8}\left[\frac{R_{2}^{4}-R_{1}^{4}}{\eta^{(2)}}+\frac{R_{1}^{4}}{\eta^{(1)}}+C^{2}\left(\frac{\epsilon^{(2)}\left(\lambda^{(2)}\right)^{2}}{3\left(\eta^{(2)}\right)^{3}}\left(R_{2}^{6}-R_{1}^{6}\right)+\frac{\epsilon^{(1)}\left(\lambda^{(1)}\right)^{2}}{3\left(\eta^{(1)}\right)^{3}} R_{1}^{6}\right)\right],
\end{aligned}
$$

and

$$
\bar{v}_{l}=\frac{Q_{l}}{\pi R_{2}^{2}}=\frac{C}{8 R_{2}^{2}}\left[\frac{R_{2}^{4}-R_{1}^{4}}{\eta^{(2)}}+\frac{R_{1}^{4}}{\eta^{(1)}}+C^{2}\left(\frac{\epsilon^{(2)}\left(\lambda^{(2)}\right)^{2}}{3\left(\eta^{(2)}\right)^{3}}\left(R_{2}^{6}-R_{1}^{6}\right)+\frac{\epsilon^{(1)}\left(\lambda^{(1)}\right)^{2}}{3\left(\eta^{(1)}\right)^{3}} R_{1}^{6}\right)\right],
$$

is the average velocity of two immiscible LPTT fluids.

\subsection{Solution for Exponential Phan-Thien-Tanner (EPTT) Model}

Constitutive equation for EPTT fluid model (8) is

$$
f\left(\operatorname{tr} \boldsymbol{T}^{(k)}\right)=\exp \left(\frac{\epsilon^{(k)} \lambda^{(k)}}{\eta^{(k)}} \operatorname{tr} \boldsymbol{T}^{(k)}\right), \quad k=1,2,
$$

with the help of expression (24), we get

$$
f\left(\operatorname{tr} \boldsymbol{T}^{(k)}\right)=\exp \left(\frac{C^{2} \epsilon^{(k)} \lambda^{(k) 2}}{2 \eta^{(k) 2}} r^{2}\right), \quad k=1,2 .
$$

Let 


$$
M^{(k)}=\frac{\epsilon^{(k)} \lambda^{(k) 2}}{2 \eta^{(k) 2}} \quad k=1,2
$$

then

$$
f\left(\operatorname{tr} T^{(k)}\right)=\exp \left(C^{2} M^{(k)} r^{2}\right), \quad k=1,2 .
$$

Substituting function (42) and shear stresses (22) in differential Equation (25), we get

$$
\frac{\mathrm{d} w_{e}^{(k)}}{\mathrm{d} r}=-\exp \left(C^{2} M^{(k)} r^{2}\right) \frac{C r}{2 \eta^{(k)}}, \quad k=1,2,
$$

where subscript $e$ is used for EPTT fluid model. Integration with respect to $r$ gives

$$
w_{e}^{(k)}=-\frac{1}{4 C M^{(k)} \eta^{(k)}} \exp \left(C^{2} M^{(k)} r^{2}\right)+E^{(k)}, \quad k=1,2,
$$

where $E^{(k)}, k=1,2$ are the constants of integration, and can be evaluated by using boundary conditions (18) and (17) for $k=1,2$. Both the velocity profiles become

$$
\begin{aligned}
w_{e}^{(1)}= & \frac{1}{4 C M^{(1)} \eta^{(1)}}\left[\exp \left(C^{2} M^{(1)} R_{1}^{2}\right)-\exp \left(C^{2} M^{(1)} r^{2}\right)\right] \\
& +\frac{1}{4 C M^{(2)} \eta^{(2)}}\left[\exp \left(C^{2} M^{(2)} R_{2}^{2}\right)-\exp \left(C^{2} M^{(2)} R_{1}^{2}\right)\right] ; \quad 0 \leq r \leq R_{1} \\
w_{e}^{(2)}= & \frac{1}{4 C M^{(2)} \eta^{(2)}}\left[\exp \left(C^{2} M^{(2)} R_{2}^{2}\right)-\exp \left(C^{2} M^{(2)} r^{2}\right)\right] ; \quad R_{1} \leq r \leq R_{2} .
\end{aligned}
$$

Flow rates of both the immiscible fluids are obtained as

$$
\begin{aligned}
Q_{e}^{(1)}= & \frac{\pi}{4 C M^{(1)} \eta^{(1)}}\left[R_{1}^{2} \exp \left(C^{2} M^{(1)} R_{1}^{2}\right)-\frac{1}{C^{2} M^{(1)}}\left\{\exp \left(C^{2} M^{(1)} R_{1}^{2}\right)-1\right\}\right] \\
& +\frac{\pi R_{1}^{2}}{4 C M^{(2)} \eta^{(2)}}\left[\exp \left(C^{2} M^{(2)} R_{2}^{2}\right)-\exp \left(C^{2} M^{(2)} R_{1}^{2}\right)\right], \\
Q_{e}^{(2)}= & \frac{\pi}{4 C M^{(2)} \eta^{(2)}}\left[-\frac{1}{C^{2} M^{(2)}}\left\{\exp \left(C^{2} M^{(2)} R_{2}^{2}\right)-\exp \left(C^{2} M^{(2)} R_{1}^{2}\right)\right\}\right. \\
& \left.+\left(R_{2}^{2}-R_{1}^{2}\right) \exp \left(C^{2} M^{(2)} R_{2}^{2}\right)\right] .
\end{aligned}
$$

Total volume flux is given by

$$
\begin{aligned}
Q_{e}= & \frac{\pi}{4 C}\left[\frac{1}{M^{(1)} \eta^{(1)}}\left\{\frac{1}{C^{2} M^{(1)}}+\left(R_{1}^{2}-\frac{1}{C^{2} M^{(1)}}\right) \exp \left(C^{2} M^{(1)} R_{1}^{2}\right)\right\}\right. \\
& +\frac{1}{M^{(2)} \eta^{(2)}}\left\{\left(R_{2}^{2}-\frac{1}{C^{2} M^{(2)}}\right) \exp \left(C^{2} M^{(2)} R_{2}^{2}\right)\right. \\
& \left.\left.-\left(R_{1}^{2}-\frac{1}{C^{2} M^{(2)}}\right) \exp \left(C^{2} M^{(2)} R_{1}^{2}\right)\right\}\right] .
\end{aligned}
$$

Average velocity $\bar{v}$ is given by 


$$
\begin{aligned}
\bar{v}= & \frac{1}{4 C R_{2}^{2}}\left[\frac{1}{M^{(1)} \eta^{(1)}}\left\{\frac{1}{C^{2} M^{(1)}}+\left(R_{1}^{2}-\frac{1}{C^{2} M^{(1)}}\right) \exp \left(C^{2} M^{(1)} R_{1}^{2}\right)\right\}\right. \\
& +\frac{1}{M^{(2)} \eta^{(2)}}\left\{\left(R_{2}^{2}-\frac{1}{C^{2} M^{(2)}}\right) \exp \left(C^{2} M^{(2)} R_{2}^{2}\right)\right. \\
& \left.\left.-\left(R_{1}^{2}-\frac{1}{C^{2} M^{(2)}}\right) \exp \left(C^{2} M^{(2)} R_{1}^{2}\right)\right\}\right],
\end{aligned}
$$

for two immiscible EPTT fluids.

\section{Special Cases}

Case \#1 If $R_{1}=0, R_{2}=R, \eta^{(k)}=\eta, \eta^{(k)}=\eta, \lambda^{(k)}=\lambda$ and $M^{(k)}=M$ for $k=1,2$, then the pressure driven two fluids reduce to a single fluid and flow becomes Poiseuile flow for PTT fluids, that is,

$$
\begin{gathered}
w_{u}=C \frac{\left(R^{2}-r^{2}\right)}{4 \eta} ; \quad 0 \leq r \leq R . \\
w_{l}=C \frac{\left(R^{2}-r^{2}\right)}{4 \eta}\left[1+\frac{M}{2} C^{2}\left(R^{2}+r^{2}\right)\right] ; \quad 0 \leq r \leq R . \\
w_{e}=\frac{1}{4 C M \eta}\left[\exp \left(M C^{2} R^{2}\right)-\exp \left(M C^{2} r^{2}\right)\right] ; \quad 0 \leq r \leq R .
\end{gathered}
$$

where $R$ represents the radius of the cylinder, $\eta$ is the viscosity of the fluid, $\epsilon$ the parameter of elongation, $\lambda$ the relaxation time, and $M=\frac{\epsilon \lambda^{2}}{2 \eta^{2}}$.

Here, $w_{u}, w_{l}$ and $w_{e}$ given by Equations (54)-(56), are the velocity profiles for pressure driven flows of UC (same as Newtonian), LPTT and EPTT fluid models, respectively.

Case \#2 If $R_{2} \rightarrow R_{1}$ then we obtain solutions for one layer UC, LPTT, and EPTT fluid models, same as given by Case \# 1 .

Case \#3 If $\lambda^{(1)}=0$ then two immiscible fluids will reduce to the cases of Newtonian-Newtonian, Newtonian-LPTT, Newtonian-EPTT immiscible fluid models.

Case \#4 If $\lambda^{(2)}=0$ then we will obtain the cases for Newtonian-Newtownian, LPTT-Newtonian, EPTT-Newtonian models, respectively.

Case \#5 If $\epsilon^{(1)}=0$ then all of the three cases will reduce to Maxwell-UC, Maxwell-LPTT, Maxwell-EPTT fluid models, respectively.

Case \#6 If $\epsilon^{(2)}=0$ then the three cases will reduce to UC-Maxwell, LPTT-Maxwell, EPTT-Maxwell fluid models, respectively.

\section{Non-Dimensionalization}

Introducing non-dimensional parameters

$$
r^{*}=\frac{r}{R_{2}}, \quad z^{*}=\frac{z}{R_{2}}, \quad \delta=\frac{R_{1}}{R_{2}}, \quad w^{(k)^{*}}=\frac{w^{(k)}}{V},
$$




$$
\lambda^{(k)^{*}}=\frac{\lambda^{(k)}}{R_{k} / V}, \quad \Re_{k}=\frac{\rho V R_{k}}{\eta^{(k)}}, \quad C^{*}=\frac{C}{\rho V^{2} / R_{2}}, k=1,2,
$$

with the assumption $\rho^{(1)}=\rho^{(2)}=\rho$, Equation (27) take the form

$$
\begin{aligned}
& w_{u}^{(1)^{*}}=\frac{C^{*}}{4}\left[\frac{\Re_{1}}{\delta}\left(\delta^{2}-r^{* 2}\right)+\Re_{2}\left(1-\delta^{2}\right)\right] ; \quad 0 \leq r \leq \delta, \\
& w_{u}^{(2) *}=\frac{C^{*}}{4} \Re_{2}\left(1-r^{* 2}\right) ; \quad \delta \leq r \leq 1 .
\end{aligned}
$$

Equation (37) and Equation (36) are transformed as

$$
\begin{aligned}
w_{l}^{(1)^{*}}= & \frac{C^{*}}{4}\left[\frac{\Re_{1}}{\delta}\left(\delta^{2}-r^{* 2}\right)+\Re_{2}\left(1-\delta^{2}\right)\right] \\
& +\frac{C^{* 3}}{16}\left[\frac{\mathfrak{R}_{1}^{3}}{\delta} M^{(1)^{*}}\left(\delta^{4}-r^{* 4}\right)+\mathfrak{R}_{2}^{3} M^{(2)^{*}}\left(1-\delta^{4}\right)\right] ; 0 \leq r \leq \delta, \\
w_{l}^{(2)^{*}}= & \frac{C^{*}}{4} \mathfrak{R}_{2}\left(1-r^{* 2}\right)\left[1+\frac{C^{* 2}}{4} \mathfrak{R}_{2}^{2} M^{(2)^{*}}\left(1+r^{* 2}\right)\right] ; \delta \leq r \leq 1 .
\end{aligned}
$$

Velocity components for EPTT fluid model becomes

$$
\begin{aligned}
w_{e}^{(1)^{*}}= & \frac{1}{2 C^{*} M^{(1)^{*}}} \frac{1}{\delta \Re_{1}}\left[\exp \left(\frac{\delta^{2} \mathfrak{R}_{1}^{2}}{2} C^{* 2} M^{(1)^{*}}\right)-\exp \left(\frac{\mathfrak{R}_{1}^{2}}{2} C^{* 2} M^{(1)^{*}} r^{* 2}\right)\right] \\
& +\frac{1}{2 C^{*} M^{(2)^{*}}} \frac{1}{\mathfrak{R}_{2}}\left[\exp \left(\frac{\mathfrak{R}_{2}^{2}}{2} C^{* 2} M^{(2)^{*}}\right)-\exp \left(\frac{\delta^{2} \mathfrak{R}_{2}^{2}}{2} C^{* 2} M^{(2)^{*}}\right)\right] ; 0 \leq r^{*} \leq \delta, \\
w_{e}^{(2)^{*}}= & \frac{1}{2 C^{*} M^{(2)^{*}}} \frac{1}{\mathfrak{R}_{2}}\left[\exp \left(\frac{\Re_{2}^{2}}{2} C^{* 2} M^{(2)^{*}}\right)-\exp \left(\frac{\mathfrak{R}_{2}^{2}}{2} C^{* 2} M^{(2)^{*}} r^{* 2}\right)\right] ; \delta \leq r^{*} \leq 1,(62)
\end{aligned}
$$

where $M^{(k)^{*}}=\frac{M^{(k)}}{R_{k}^{2} / \eta^{(k) 2} V^{2}}$.

\section{Graphical Results and Discussion}

In this paper, the exact solution of the two layer non-Newtonian fluid through a horizontally placed cylindrical pipe is obtained and derived mathematical results are discussed and graphed.

In this section, graphs demonstrating the behavior of the various parameters in conjunction with the velocity profile are presented.

Figure 2 is showing the effect of viscosity (i.e., Reynolds number $\mathfrak{R}_{i}, i=1,2$ ) on velocity profile for UCM fluid model. It can be noted from Figure 2 (a) that the viscosity of the outer layer effects the whole region, but the viscosity of inner fluid layer (Figure 2(b)) only effect its own region, which is true for all three types of fluid models. It is also observed that as the viscosity of the fluid increases, the fluid becomes thick and its velocity decreases, as expected physically.

Figure 3 is showing the effect of pressure gradient on velocity profile for UCM fluid model. With the increase in pressure velocity is increased but the thickening effect can be noticed easily. 


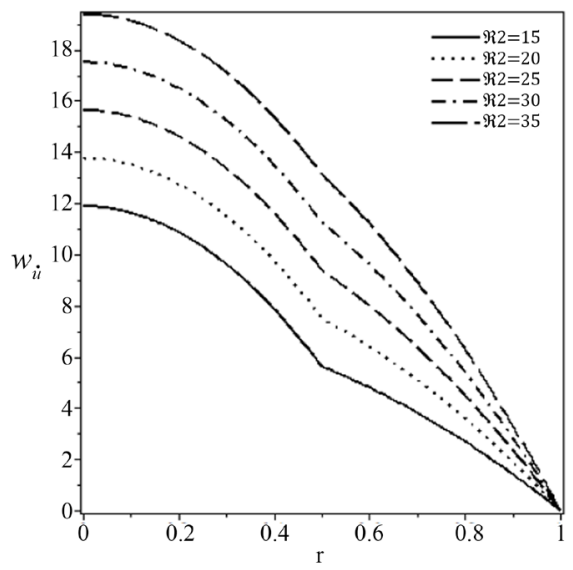

(a)

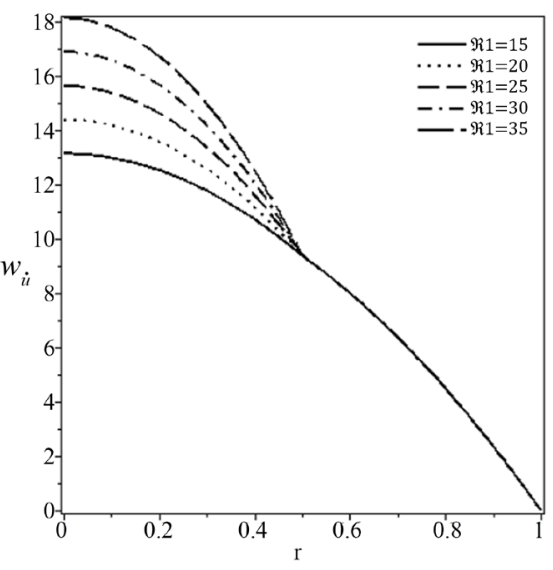

(b)

Figure 2. The effect of (a) $\mathfrak{R}^{(2)}$ keeping $\mathfrak{R}^{(1)}=25$ and (b) $\mathfrak{R}^{(1)}$ keeping $\mathfrak{R}^{(2)}=25$ on velocity profile of UCM fluid model when $C=2, \delta=0.5$.

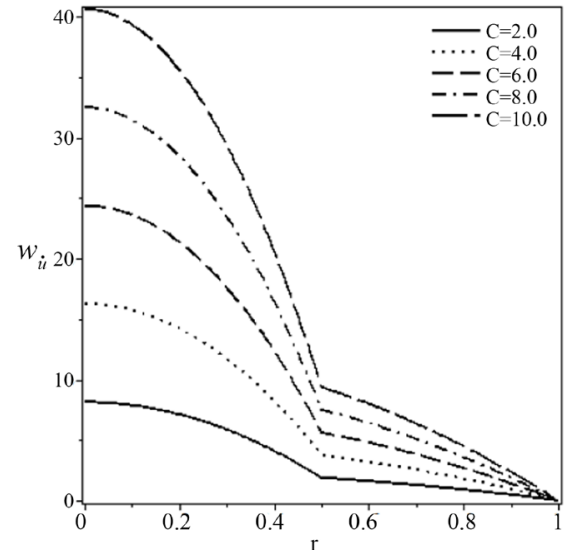

(a)

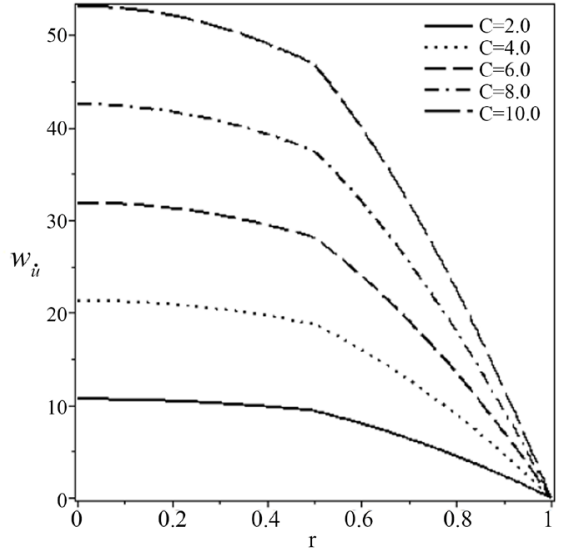

(b)

Figure 3. The effect of pressure gradient $C$ keeping (a) $\Re^{(1)}=25$ and $\Re^{(2)}=5$ $\mathfrak{R}^{(1)}=5$ and $\mathfrak{R}^{(2)}=25$ on velocity profile of UCM fluid model when $\delta=0.5$.

Figure 4 and Figure 5 are showing the effect of viscosity on velocity profile for LPTT fluid model. In Figure 4 an increase in velocity profile can be observed with the increase in relaxation parameter.

Figure 5 shows an elongation effect, $\epsilon^{(2)}$ on velocity profile. When the effect of $\epsilon^{(2)}$ and $\lambda^{(2)}$ are combined, a huge effect can be noticed.

In Figure 6 and Figure 7 the effect of viscosity on velocity profile for EPTT fluid model can be observed. Figure 6 depicts how relaxation parameter is affecting the velocity profile. With increase in relaxation parameter, velocity is increased. From Figure 7, it can be observed that elongation alone has very little influence on the velocity profile, but when it is combined with the relaxation parameter, velocity of EPTT fluid has increased significantly.

Observing Figure 2(a), Figure 4(a) and Figure 6(a), we find that for small values of elongation and relaxation parameters, all three PTT fluids have almost the same behavior, but with increasing these two parameters LPTT and EPTT 

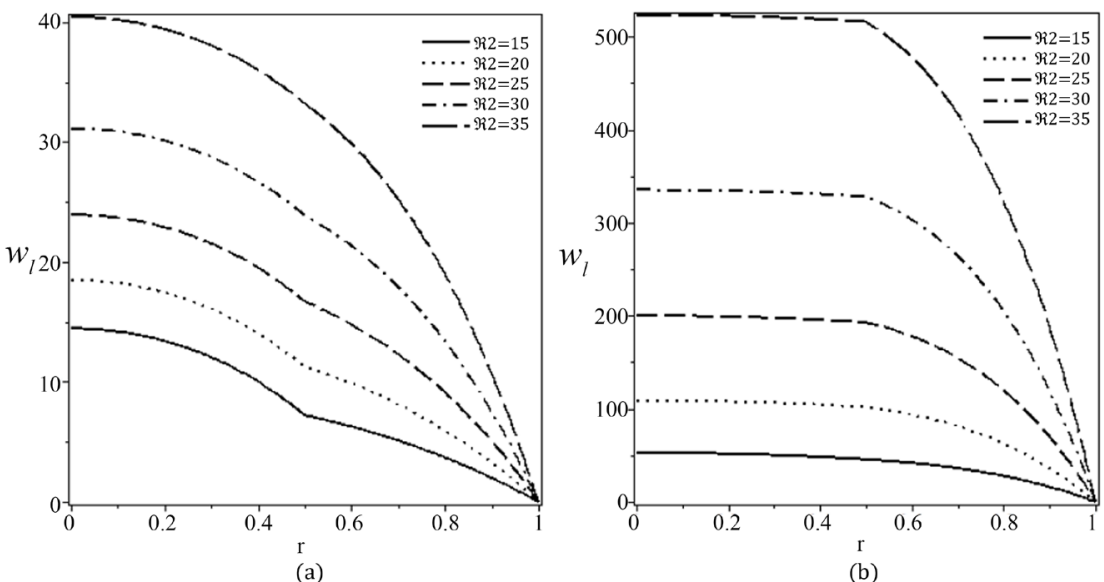

Figure 4. The effect of $\mathfrak{R}^{(2)}$ keeping (a) $\lambda^{(2)}=0.1$ (b) $\lambda^{(2)}=0.5$, on velocity profile of LPTT fluid model when $\mathfrak{R}^{(1)}=25, \epsilon^{(1)}=0.1, \epsilon^{(2)}=0.1, \lambda^{(1)}=0.1, C=2, \delta=0.5$.
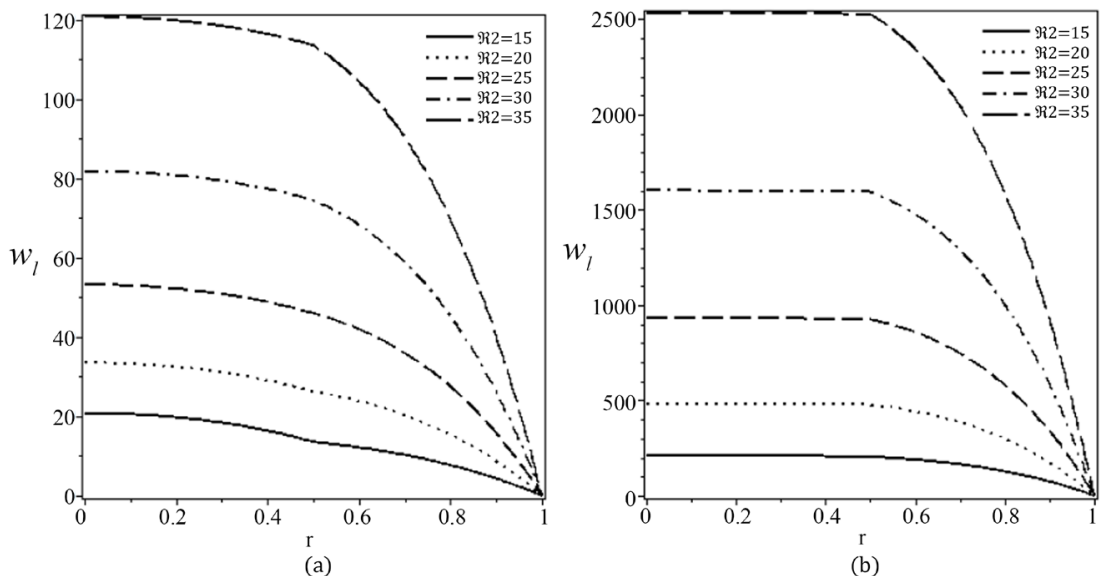

Figure 5. The effect of $\mathfrak{R}^{(2)}$ on velocity profile of LPTT fluid model keeping (a) $\lambda^{(2)}=0.1$ and $\epsilon^{(2)}=0.5 \quad$ (b) $\lambda^{(2)}=0.5$ and $\epsilon^{(2)}=0.5$ when $\mathfrak{R}^{(1)}=25, \epsilon^{(1)}=0.1$, $\lambda^{(1)}=0.1, \quad C=2, \delta=0.5$.
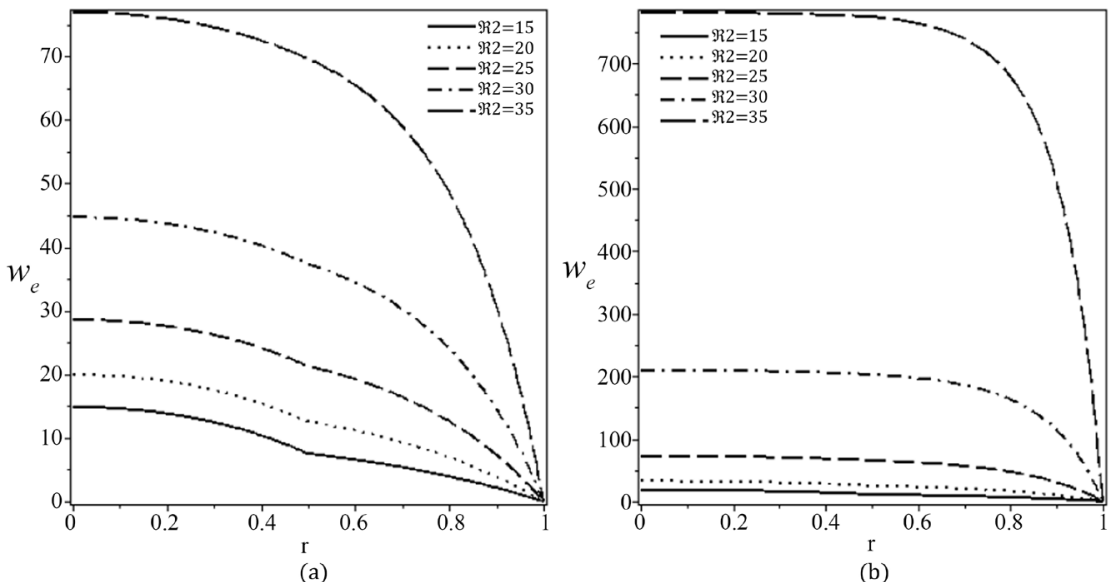

Figure 6. The effect of $\mathfrak{R}^{(2)}$ keeping (a) $\lambda^{(2)}=0.1$ (b) $\lambda^{(2)}=0.15$ on velocity profile of EPTT fluid model when $\mathfrak{R}^{(1)}=25, \lambda^{(1)}=0.1, \epsilon^{(1)}=0.1, \epsilon^{(2)}=0.1, C=2, \delta=0.5$. 


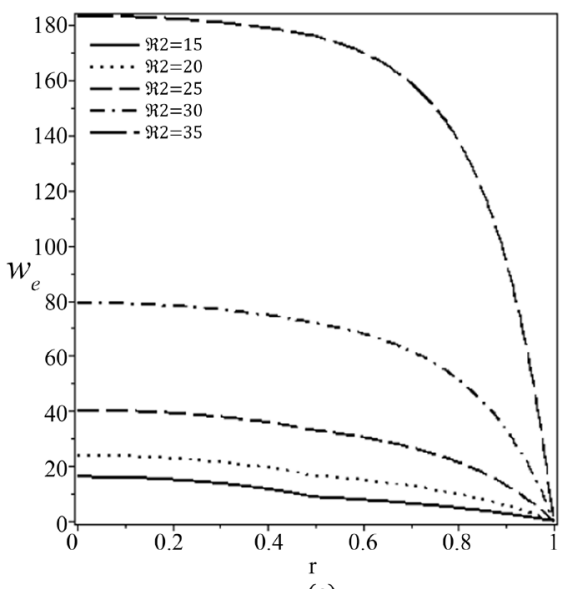

(a)

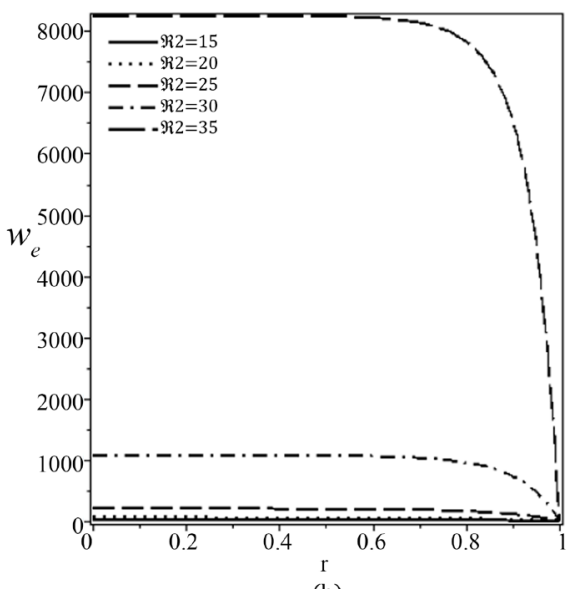

(b)

Figure 7. The effect of $\mathfrak{R}^{(2)}$ keeping (a) $\lambda^{(2)}=0.1, \epsilon^{(2)}=0.15$ (b) $\lambda^{(2)}=0.15$, $\epsilon^{(2)}=0.15$ on velocity profile of EPTT fluid model when $\mathfrak{R}^{(1)}=25, \lambda^{(1)}=0.1$, $\epsilon^{(1)}=0.1, \quad C=2, \delta=0.5$.

fluid model are showing thinning effect (Figure 4(b) and Figure 6(b)). EPTT fluid becomes thinner than LPTT fluid for the same values of $\lambda$ and $\epsilon$.

\section{Conclusions}

To summarize the results of our analysis of the pressure driven flow of two immiscible non-Newtonian fluids in a pipe by focusing our attention on three fluid models known as UCM, LPTT and EPTT, we present visual evidence reflecting the behavior of the various parameters in conjunction with the velocity profile.

- For all the three fluid models, the viscosity of fluid $k=(1)$, in the region $0 \leq r<\delta$ does not effect the flow of the fluid $k=(2)$, in the region $\delta<r \leq 1$. Thus, the flow parameters of fluid (2) are independent of the viscosity of fluid (1).

- Viscosity of fluid (1) is affected by the flow parameters (velocities and flow rates) of fluid (1) and fluid (2).

- Increase in pressure gradient, elongation and relaxation time parameters enhance the speed of flow, showing the thinning effect.

- For fixed value of parameters we compare the velocities as follows

$$
w_{u}>w_{l}>w_{e}
$$

where $w_{u}, w_{l}$ and $w_{e}$ are the velocities of the UCM, LPTT and EPTT fluid models, respectively.

- We recover the Newtonian case from LPTT and EPTT fluid models as $\lambda \rightarrow 0$.

\section{Acknowledgements}

The authors greatly acknowledge the financial support by the Penn State York Campus to publish this work. 


\section{Conflicts of Interest}

The authors declare no conflicts of interest regarding the publication of this paper.

\section{References}

[1] Bird, R.B., Armstrong, R.C. and Hassager, O. (1987) Dynamics of Polymeric Liquids. In: Vol. 1 Fluid Mechanics, 2nd Edition, John Wiley and Sons, Inc., Hoboken, New Jersey.

[2] Phan-Thien, N. and Tanner, R.I. (1977) A New Constitutive Equation Derived from Network Theory. Journal of Non-Newtonian Fluid Mechanics, 2, 353-365. https://doi.org/10.1016/0377-0257(77)80021-9

[3] Tanner, R.I. (2000) Engineering Rheology. Clarendon Press, Oxford.

[4] Oliveira, P.J. and Pinho, F.T. (1999) Analytical Solution for the Fully-Developed Channel and Pipe Flow of Phan-Thien-Tanner Fluids. Journal of Fluid Mechanics, 387, 271-280. https://doi.org/10.1017/S002211209900453X

[5] Pinho, F.T. and Oliveira, P.J. (2000) Analysis of Forced Convection in Pipes and Channels with Simplified Phan-Thien-Tanner Fluid. International Journal of Heat and Mass Transfer, 43, 2273-2287. https://doi.org/10.1016/S0017-9310(99)00303-8

[6] Pinho, F.T. and Oliveria, P.J. (2000) Axial Annular Flow of a Nonlinear Viscoelastic Fluid: An Analytical Solution. Journal of Non-Newtonian Fluid Mechanics, 93, 325-337.

[7] Alves, M.A., Pinho, F.T. and Oliveira, P.J. (2001) Study of Steady Pipe and Channel Flows of Single-Mode Phan-Thien-Tanner Fluid. Journal of Non-Newtonian Fluid Mechanics, 101, 55-76. https://doi.org/10.1016/S0377-0257(01)00159-8

[8] Letelier, M.F. and Siginer, D.A. (2005) On the Fully Developed Tube Flow of a Class of Non-Linear Viscoelastic Fluids. International Journal of Non-Linear Mechanics, 40, 485-493. https://doi.org/10.1016/j.ijnonlinmec.2004.07.009

[9] Siddiqui, A.M., Mahmood, R. and Ghori, Q.K. (2006) Some Exact Solutions for the Thin Film Flow of a PTT Fluid. Physics Letters A, 356, 353-356.

https://doi.org/10.1016/j.physleta.2006.03.071

[10] Packham, B.A. and Shall, R. (1971) Stratified Laminar Flow of Two Immiscible Fluids. Mathematical Proceedings of the Cambridge Philosophical Society, 69, 443-448. https://doi.org/10.1017/S0305004100046880

[11] Brauner, N. (1991) Two-Phase Liquid-Liquid Annular Flow. International Journal of Multiphase Flow, 17, 59-76. https://doi.org/10.1016/0301-9322(91)90070-J

[12] Kapur, J.N. and Shukla, J.B. (1964) The Flow of Incompressible Immiscible Fluids between Two Plates. Applied Scientific Research, Section A, 13, 55-60. 